\title{
DNA-carbon nano onion aggregate: triangle, hexagon, six-petal flower to dead-end network
}

\author{
Dipak Gorakh Babar ${ }^{1} \cdot$ Bholanath Pakhira $^{2,3} \cdot$ Sabyasachi Sarkar $^{3}$ (D)
}

Received: 28 March 2017/ Accepted: 11 May 2017/Published online: 17 May 2017

(C) The Author(s) 2017. This article is an open access publication

\begin{abstract}
The interaction between calf-thymus (CT) dsDNA and water soluble carbon nano onion (wsCNO) in water follows denaturation of dsDNA (double stranded) to ssDNA (single stranded) as monitored by optical spectroscopy. The ssDNA concomitantly wraps the spiky surface of wsCNO to create triangular aggregate as the building block as observed by time-dependent SEM images. These triangles further aggregate leading to six-petal flower arrangement via hexagon and finally reach a dead end network as imaged by SEM and optical fluorescence microscopy. The dead-end network aggregate lost the intrinsic optical property of DNA suggesting complete loss of its activity.
\end{abstract}

Keywords Calf thymus DNA · wsCNO - Self-assembly · Six-petal flower aggregate $\cdot$ Dead-end network

\section{Introduction}

Several short single-stranded (ss) DNA interact with a long single-stranded (ss) DNA to generate different shapes such like squares, triangles, and five-pointed stars.

Sabyasachi Sarkar

abya@iitk.ac.in; sabby@chem.iiests.ac.in

1 Department of Chemistry, Indian Institute of Technology, Kanpur, Uttar Pradesh 208016, India

2 Department of Chemistry, Sister Nibedita Govt. General Degree College for Girls, Hastings House, Alipore, Kolkata, West Bengal 700027, India

3 Nano Science and Synthetic Leaf Laboratory at Downing Hall, Center for Healthcare Science and Technology, Indian Institute of Engineering Science and Technology, Shibpur, Botanic Garden, Howrah, West Bengal 711013, India
Such scaffolded DNA origami is driven by the creation of novel biomaterials for analytical and therapeutic purposes. This type of exotic ssDNA-ssDNA interaction is exclusively bio-molecular in nature in the presence of $\mathrm{Mg}^{2+}$ and other ions present in buffer used in such study (Andersen et al. 2009; Ke et al. 2012; Rothemund 2006). Using graphene surface as template sort stranded ssDNA aggregates in spherical shapes and in elongated network (Akca et al. 2011). However, the interaction between ssDNA and single-wall carbon nano tube (CNT) constitutes DNA-carbon-nano tube hybrid (DNA-CNT) where the CNT was wrapped with a self-assembled coating of ssDNA (Johnson et al. 2010). There are several reports of fluorescing nano carbon dots to image different cell lines. Such studies are generally made use of directly using the nano carbon produced without knowing the effective amount used in those experiments (Bourlinos et al. 2008; Sun et al. 2006; Wang et al. 2010; Yang et al. 2009). The dose-related toxicity-non toxicity issue of the nano carbon used in such cell line imaging has not been addressed. Use of water-soluble carbon nano onion (wsCNO) in known amount is shown to image the entire life cycle of Drosophila melanogaster (Ghosh et al. 2011). In addition, the dose-dependent non-toxic and toxic use of wsCNO has also been reported in imaging the life cycle and controlling the population of mosquito, respectively (Saxena et al. 2013). In the plant system there has been a report for an optimum concentration threshold for the beneficial use of wsCNO (Saxena et al. 2014). These reports clearly suggest that there is a concentration window in the use of nano carbon and exceeding the limit it has deleterious effect to biological systems. As DNA is the most important bio-molecule that controls the structure and function of the cell, it is thus important to check the effect of wsCNO directly on dsDNA. Therefore, we

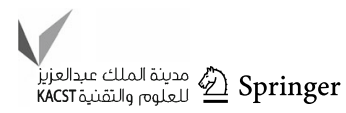



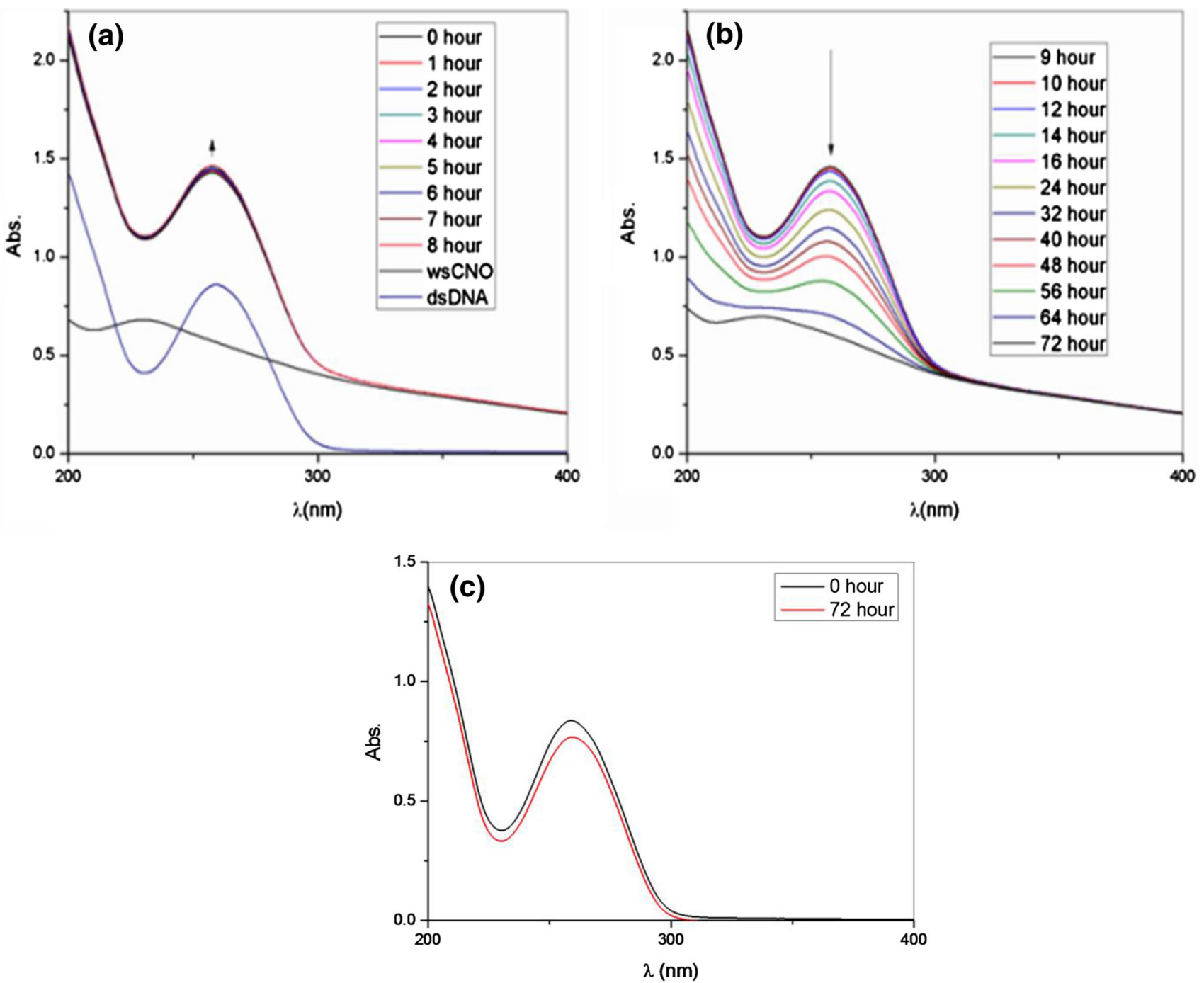

Fig. 1 a Time-dependent spectral change of CT-DNA with wsCNO in water in the initial period of rise in the $260 \mathrm{~nm}$ absorption till $8 \mathrm{~h}$ as labeled in the inset. Black trace is the electronic spectrum of wsCNO and blue trace is the electronic spectrum of CT-DNA. b The

report the interaction between calf-thymus (CT) dsDNA with water-soluble carbon nano onion (wsCNO) in water. We show that wsCNO first unzipped dsDNA to ssDNA and the ssDNA thus formed wrapped the wsCNO present in the solution leading to several macromolecular aggregation starting with finite shapes like triangle, polyhedron, flower petals, six-petal flowers, and finally to an extended dead-end network structure.

\section{Experimental}

\section{Materials}

Calf thymus DNA (CT-DNA) was purchased from Sigma Aldrich (absorbance ratio A260/A280 = 1.8). The water- gradual fall of $260 \mathrm{~nm}$ absorption band with time as labeled in the inset. c Electronic spectra of CT-DNA solution on standing for $72 \mathrm{~h}$ showed slight decay retaining $\sim 91 \%$ of its absorption profile

soluble CNOs were synthesized using previously reported methods (Ghosh et al. 2011; Sonkar et al. 2012). Water from a Millipore Milli-Q system (conductivity $\leq 0.1 \mu \mathrm{S} \mathrm{cm}^{-1}$ ) has been used.

\section{Macroscopic and spectroscopic characterization}

Electronic spectral measurements were carried out with JASCO, V-630 spectrophotometer. For optical fluorescence microscopy, slides were examined using an inverse microscope (Axiovert $200 \mathrm{M}$, Zeiss equipped with Axio Cam and AxioVision software) with FITC green filter. Solution $\mathrm{pH}$ were measured using Systronics Digital $\mathrm{pH}$ meter 335 and for scanning electron microscopy (FESEM), a SUPRA 40VP field-emission SEM (Carl Zeiss NTS $\mathrm{GmbH}$, Oberkochen, Germany) equipped with an energy- 
Fig. 2 Images of DNA composite; a 1 h: DNAwsCNO, b 4 h: growth, $\mathbf{c} 8$ h: aggregation, d $16 \mathrm{~h}$ : triangle formation, e hexagon, $\mathbf{f}$ flower, $\mathbf{g}$ assorted flowers, $\mathbf{h}$ selected six-petal flower
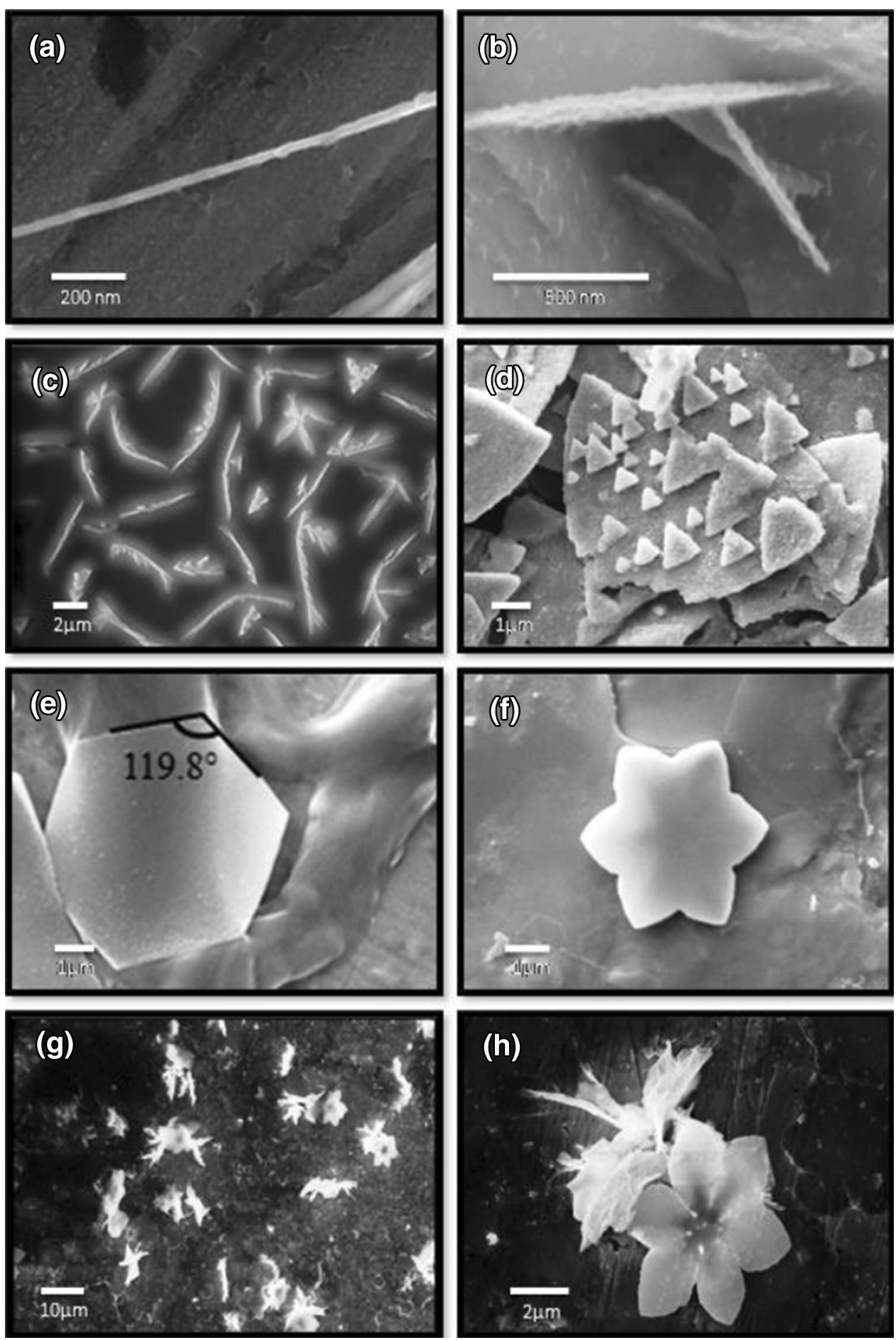

dispersive X-ray (EDX) unit, in high-vacuum mode operated at $10 \mathrm{kV}$, was used for the visualization of the size and morphology of CT-DNA-wsCNO composite.

\section{Preparation of CT-DNA-wsCNOs composite}

Calf thymus DNA (CT-DNA) (Sigma Aldrich, absorbance ratio $\mathrm{A} 260 / \mathrm{A} 280=1.8$ ) and the $\mathrm{wSCNOs}^{5}$ were dissolved in water maintained at $0{ }^{\circ} \mathrm{C}$ (Millipore Milli-Q system, conductivity $\leq 0.1 \mu \mathrm{S} \mathrm{cm}^{-1}$ ). The concentration $86.66 \mu \mathrm{g} /$ $\mathrm{ml}$ of CT-DNA and $33.32 \mu \mathrm{g} / \mathrm{ml}$ of wsCNO were used and when such DNA and wsCNOs solutions were mixed in 1:1 ratio, the $\mathrm{pH}$ of the resultant solution became 7.0. This stoichiometric ratio between CT-DNA and wsCNO is subsequently used in all experiments. The composite mixture was stored in the temperature range $1-5{ }^{\circ} \mathrm{C}$ and all

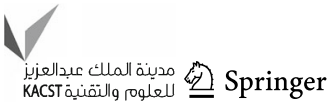




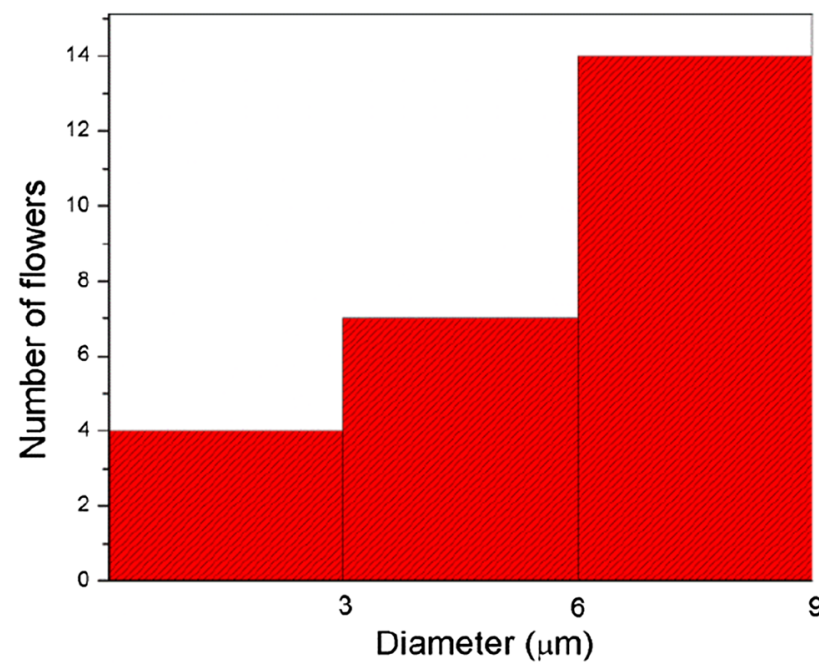

Fig. 3 Histogram: size distribution of flowers (diagonal petal-petal, data from Fig. $2 \mathrm{~g}$ )

the experiments were carried out at a temperature of around $20{ }^{\circ} \mathrm{C}$.

\section{Results and discussion}

As prepared the composite solution was used for successive analysis. Such a solution was first subjected to electronic spectroscopy monitoring the change in absorbance at $260 \mathrm{~nm}$ with time. On standing for $72 \mathrm{~h}$. around a temperature in the range $1-5{ }^{\circ} \mathrm{C}$ (fridge) the solution remains clear with no precipitation as checked by centrifugation.

The hyperchromism of the CT-DNA solution reached maximum till ca $8 \mathrm{~h}(\sim 14 \%$ increase $)$ and then its intensity gradually started decreasing. After $64 \mathrm{~h}$ most of the intensity measured at $260 \mathrm{~nm}$ absorption band is lost and after $72 \mathrm{~h}$ it completely disappeared. The spectral trace after $72 \mathrm{~h}$ clearly retains the absorption signature of wsCNO at $230 \mathrm{~nm}$ which was masked under the strong back ground absorption of DNA at the earlier stages of the mixture. Around 9\% degradation of CT-DNA was monitored by following the decrease in the intensity of $260 \mathrm{~nm}$ band under controlled conditions after $72 \mathrm{~h}$ ( Fig. 1c). The complete loss of this characteristic absorption on the addition of wsCNO in this time frame suggests that wsCNO completely damage DNA initiating its aggregation leading to unnatural chain aggregates. The rise in the intensity of the absorption band at $260 \mathrm{~nm}$ of CT-DNA (dsDNA) in the presence of wsCNO in the initial period is related to its denaturation to ss-DNA. It is known that the electronic absorption profile of ssDNA is identical to that of dsDNA with the variation in increase in the intensity of absorption (Berg et al. 2002; Wang and Son 2013). When the bases of dsDNA got completely un-stacked, the wavelength of maximum absorbance at $260 \mathrm{~nm}$ does not change but for the total conversion from dsDNA to ssDNA the absorption increases by $37 \%$ (Berg et al. 2002). However, under wsCNO only $14 \%$ increase of this intensity at the initial phase is noticed and this intensity remained constant in the initial time period between 4 and $8 \mathrm{~h}$. Therefore, in the present case it is around $38 \%$ denaturation of dsDNA to ssDNA that can be monitored and this concentration remained unchanged for $8 \mathrm{~h}$. We interpret this phenomenon as the spherical wsCNO possessing several carboxylic and hydroxyl groups initially interacting to unzip the CT-DNA (dsDNA) to ssDNA. Once this process sets in, a follow-up reaction by the separated ssDNA started to wrap the wsCNO using hydrogen bond and other conventional forces. Such interactions may cause significant structural changes of ssDNA with the loss in its characteristic absorption at $260 \mathrm{~nm}$. Once the speed in wrapping exceeded the denaturation of ds-DNA to ssDNA the characteristic DNA absorption peak at $260 \mathrm{~nm}$ continuously loses its intensity as monitored in the subsequent time-dependent absorption spectra (Fig. 1b). The denaturation rate from dsDNA to ssDNA may be slower than its wrapping by wsCNO kinetics which apparently prevents to display further increase in the intensity of the $260 \mathrm{~nm}$ peak at a later stage. The time-dependent response of the electronic spectra is shown in Fig. 1. Figure 1a shows the initial rise in the absorption of DNA due the denaturation of dsDNA to ssDNA. However, after the initial build up of $38 \%$ ssDNA within $8 \mathrm{~h}$ it started to react more rapidly with wsCNO as shown by the time-dependent subsequent electronic spectral scan from 9 to 72 h (Fig. 1b). The scan after $72 \mathrm{~h}$ showed the complete disappearance of the signature band at $260 \mathrm{~nm}$ associated with DNA. Under identical conditions the stability of CT DNA was measured as control to show that only $\sim 9 \%$ of its intensity is lost retaining 91\% DNA intact (Fig. 1c).

\section{SEM and fluorescence optical microscopy}

Based on the electronic spectral time frame we imaged the mixture of CT-DNA and wsCNO by SEM and also by fluorescence optical microscopy to understand the nature of such interaction visually.

The hydrophilic groups like $-\mathrm{COOH}$ and $-\mathrm{OH}$ attached to the outer shell of wsCNOs may interact directly with unzipped ssDNA and may also help to unzip dsDNA competing with hydrogen bonding and/or van der Waals interaction. The SEM images of sample mixture are shown from the initial mixing till $16 \mathrm{~h}$ in Fig. 2. In the beginning (Fig. 2a) the image may relate to a dsDNA with surface interaction of wsCNO. After $4 \mathrm{~h}$ (Fig. 2b) the aggregation at several places were observed and between 8 and $16 \mathrm{~h}$ such interaction clearly developed branched and designed 
Fig. 4 Fluorescence microscopy images with time: a $1 \mathrm{~h}, \mathbf{b} 4 \mathrm{~h}, \mathbf{c} 16 \mathrm{~h}, \mathbf{d} 24 \mathrm{~h}$, e $48 \mathrm{~h}, \mathbf{f} 72 \mathrm{~h}$
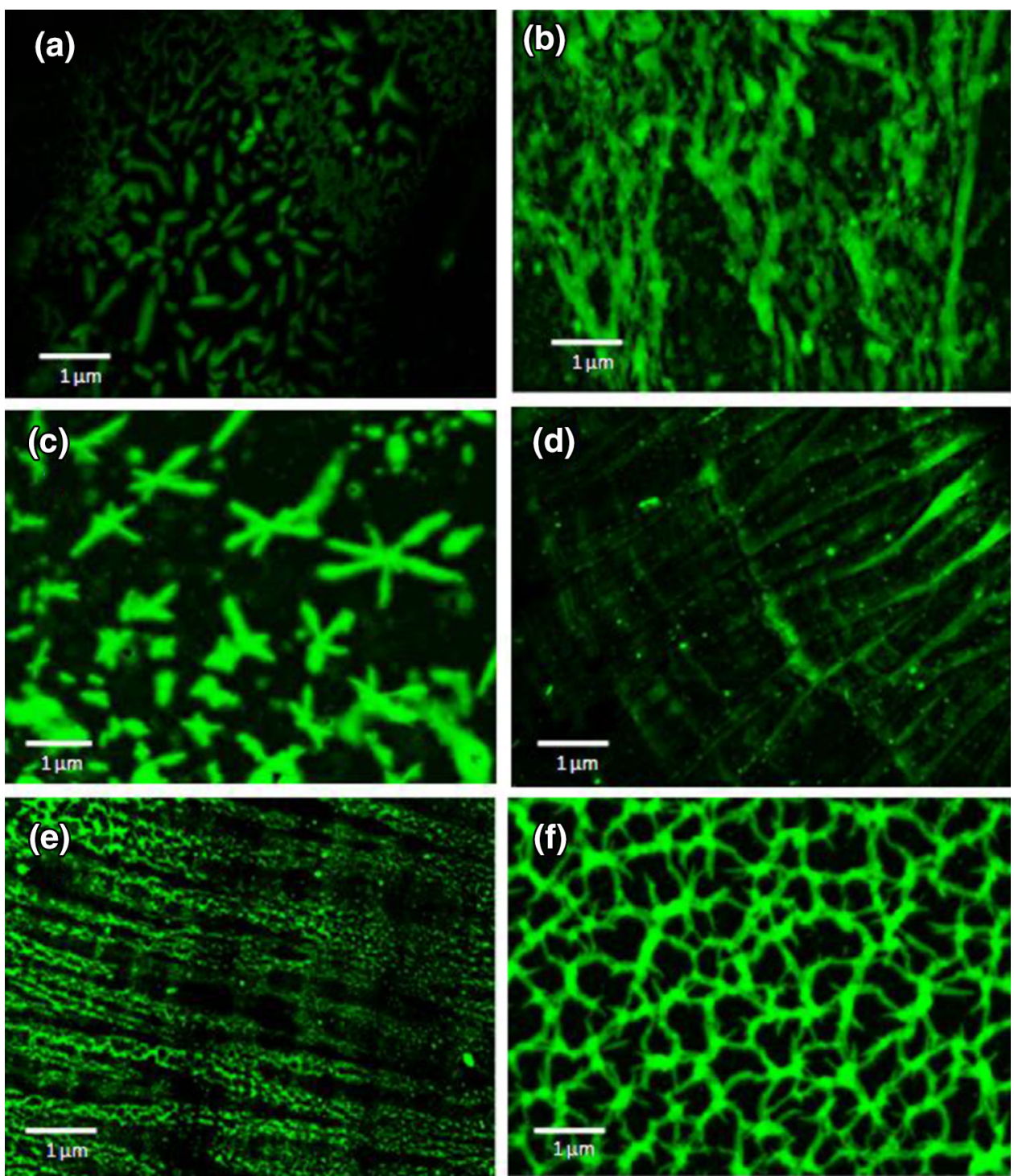

structure aggregation (Fig 2c, d). Fig. 2e-h shows the aggregation pattern where Fig. $2 \mathrm{~h}$ is a zoomed image of a six-petal structure similar to a flower. At this point the absorption of DNA at $260 \mathrm{~nm}$ started to lose its intensity suggesting the loss of known structure of DNA. Figure 2d shows the predominant shape of the composite like triangle after $16 \mathrm{~h}$. These triangles aggregate to create hexagon (angle of $119.8^{\circ}$ ) as shown in Fig. 2e. Peripheral attachment of these triangles to a hexagon may lead to six-petal design of flower as in Fig. 2f. Figure $2 \mathrm{~g}$ shows assorted sizes of such flower-shape structure. A histogram (Fig. 3) shows the size distribution pattern of such structures where large flowers with diagonal petal-petal distribution of $9 \mu \mathrm{m}$ are observed. A representative six-petal shaped flower esthetically pleasing like natural flower is shown in
Fig. $2 \mathrm{~h}$. The association of fluorescing wsCNO in such aggregation process is followed by imaging the mixture with the progress in reaction time. Figure 4 displays the fluorescence images of the composite in different time intervals. In the beginning hour (Fig. 4a), the aggregation started and became extensive during $4 \mathrm{~h}$ (Fig. 4b). At $16 \mathrm{~h}$ the different shapes with the aggregation like six-petal flowers are visible (Fig. 4c). The interesting part here is to image the aggregate in the next 24,48 and $72 \mathrm{~h}$, respectively. Figure $4 \mathrm{~d}-\mathrm{f}$ clearly shows the progress in aggregation that ended with dead-end extended network structure. It is to be noted that the time period for such aggregation using SEM or fluorescence study is around $16 \mathrm{~h}$. The electronic spectral study shows the DNA signature till $64 \mathrm{~h}$ with very low intensity This suggests that the SEM matrix 

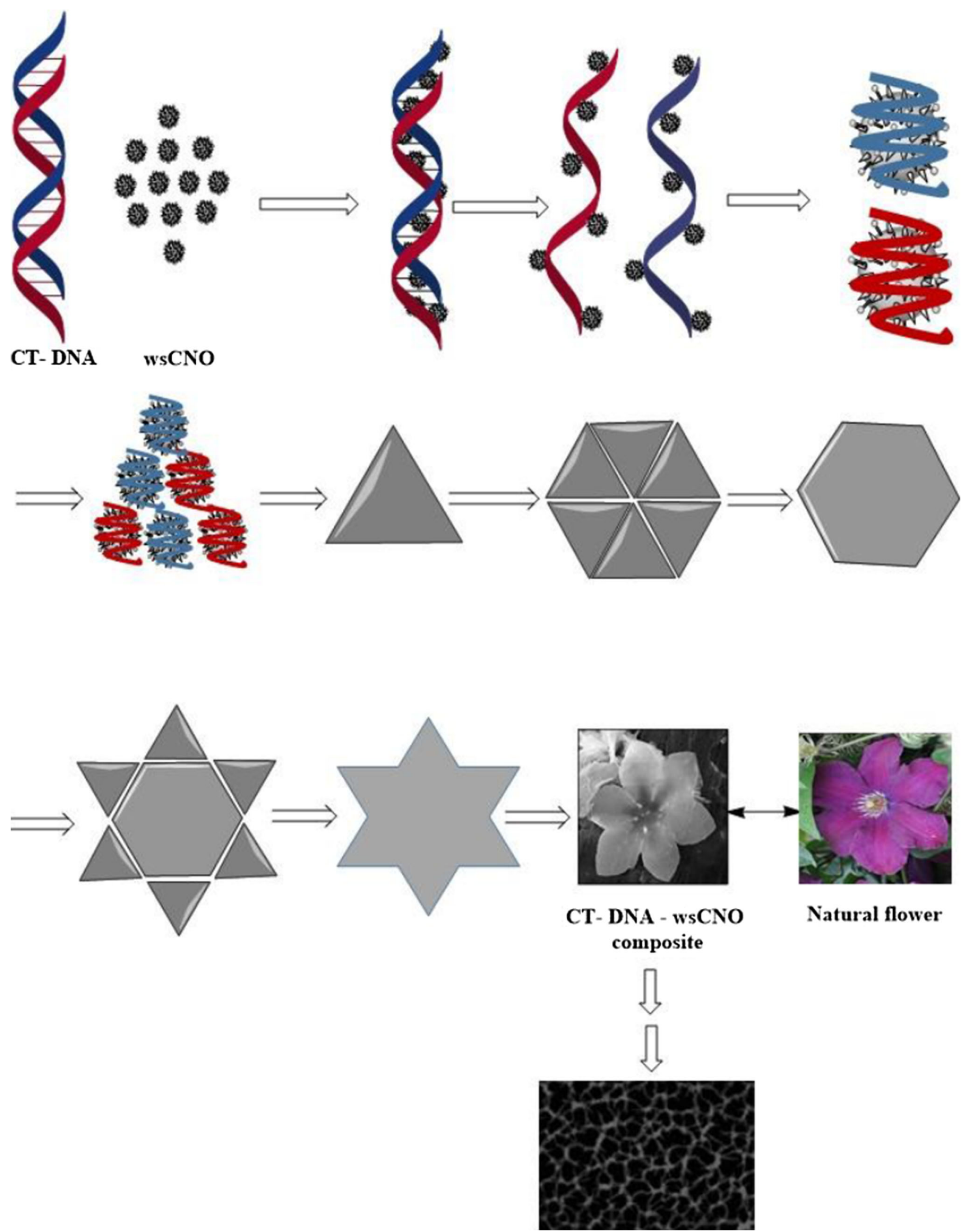

Fig. 5 The formation of flower shape composite of wsCNOs and CT-DNA with triangle and hexagon as intermediates finally to dead-end network structure 
or the microscopic slide may accelerate aggregation, especially when the water gets dried up with time (Mitra and Sarkar 2016). The possible process from denaturation to petal shape followed with the flower type growth formation is schematically shown in Fig. 5. The spontaneous formation of triangle-shaped aggregate at the initial stage may be due to the shape-related thermodynamic drive to acquire stability which further led to create stable hexagon structure.

\section{Conclusion}

In conclusion, wsCNOs is shown to unzipp CT-DNA in water to ssDNA. Once appreciable ssDNA is available, the spherical wsCNO spontaneously wraps ssDNA by its peripheral carboxylic acid and hydroxyl groups used as spikes. The assembly of this wrapped species leads to the formation of triangle, hexagon and flower shapes with several micrometers in sizes. Finally, at the end these regular structures may be driven to more stable network structure with the creation of extended dead-end network structure losing normal characteristics of DNA. As wsCNO is shown to be dose-dependent non-toxic (Ghosh et al. 2011; Saxena et al. 2013, 2014) it will be challenging to explore the interaction of wsCNO with rouged DNA to control their unwanted proliferation in certain cell growth and preventing such viral DNA replication in well-known diseases.

Acknowledgements DB and BP acknowledge CSIR, New Delhi, India for Senior Research Fellowships. SS thanks SERB-DST(SR/S1/ RFIC-01/2011 and EMR/2015/001328), New Delhi, India for sustained finanical support.

Open Access This article is distributed under the terms of the Creative Commons Attribution 4.0 International License (http:// creativecommons.org/licenses/by/4.0/), which permits unrestricted use, distribution, and reproduction in any medium, provided you give appropriate credit to the original author(s) and the source, provide a link to the Creative Commons license, and indicate if changes were made.

\section{References}

Akca S, Foroughi A, Frochtzwajg D, Postma HWC (2011) Competing interactions in DNA assembly on graphene. PLoS One 6:e18442. doi:10.1371/journal.pone. 0018442

Andersen ES et al (2009) Self-assembly of a nanoscale DNA box with a controllable lid. Nature 459:73-76. doi:10.1038/nature07971

Berg JM, Tymoczko JL, Stryer L (2002) Biochemistry, 5th edn. W. H. Freeman and Company, New York

Bourlinos AB, Stassinopoulos A, Anglos D, Zboril R, Georgakilas V, Giannelis EP (2008) Photoluminescent carbogenic dots. Chem Mater 20:4539-4541. doi:10.1021/cm800506r

Ghosh M, Sonkar SK, Saxena M, Sarkar S (2011) Carbon nanoonions for imaging the life cycle of drosophila melanogaster. Small 7:3170-3177. doi:10.1002/smll.201101158

Johnson RR, Johnson ATC, Klein ML (2010) The nature of DNAbase-carbon-nanotube interactions. Small 6:31-34. doi:10.1002/ smll.200901481

Ke Y, Voigt NV, Gothelf KV, Shih WM (2012) Multilayer DNA origami packed on hexagonal and hybrid lattices. J Am Chem Soc 134:1770-1774. doi:10.1021/ja209719k

Mitra J, Sarkar S (2016) Substrate induced morphology in a hydrosulfide-molybdenum complex. New J Chem 40:8905-8910. doi:10.1039/C6NJ01910B

Rothemund PWK (2006) Folding DNA to create nanoscale shapes and patterns. Nature 440:297-302. doi:10.1038/nature04586

Saxena M, Sonkar SK, Sarkar S (2013) Water soluble nanocarbons arrest the growth of mosquitoes. RSC Adv 3:22504-22508. doi:10.1039/C3RA44100H

Saxena M, Maity S, Sarkar S (2014) Carbon nanoparticles in 'biochar' boost wheat (Triticum aestivum) plant growth. RSC Adv 4:39948-39954. doi:10.1039/C4RA06535B

Sonkar SK, Roy M, Babar DG, Sarkar S (2012) Water soluble carbon nano-onions from wood wool as growth promoters for gram plants. Nanoscale 4:7670-7675. doi:10.1039/C2NR32408C

Sun Y-P et al (2006) Quantum-sized carbon dots for bright and colorful photoluminescence. J Am Chem Soc 128:7756-7757. doi:10.1021/ja062677d

Wang X, Son A (2013) Effects of pretreatment on the denaturation and fragmentation of genomic DNA for DNA hybridization. Environ Sci Process Impacts 15:2204-2212. doi:10.1039/ C3EM00457K

Wang X et al (2010) Bandgap-like strong fluorescence in functionalized carbon nanoparticles. Angewandte Chemie Int Ed 49:5310-5314. doi:10.1002/anie.201000982

Yang S-T et al (2009) Carbon dots for optical imaging in vivo. J Am Chem Soc 131:11308-11309. doi:10.1021/ja904843x 